\title{
Recurrent myocardial infarction with non-obstructive coronary arteries in the course of an angiographically non-obstructive, but physiologically significant, proximal left artery descending lesion recognized with fractional flow reserve
}

\author{
Michał Proczka, Adam Trzciński, Anna Skrobisz, Łukasz Kalińczuk, Janina Stępińska, Marcin Demkow \\ Department of Coronary and Structural Heart Diseases, Institute of Cardiology, Warsaw, Poland
}

Adv Interv Cardiol 2018; 14, 2 (52): 202-203

DOI: https://doi.org/10.5114/aic.2018.76415

In up to $14 \%$ of cases of acute coronary syndrome (with either elevated or non-elevated ST), contemporary high-resolution digital angiography does not identify obstructive coronary artery disease, with visually estimated \% diameter stenosis (DS) of pictured lesions of $<50 \%$. Interestingly, a novel pathophysiological concept of acute coronary syndrome in the course of non-obstructive coronary artery disease has been introduced recently (myocardial infarction with non-obstructive coronary arteries - MINOCA). We present a 52-year-old male smoker, who was admitted after sudden cardiac arrest following ventricular fibrillation. Remarkably, the patient had a history of anterior ST elevation myocardial infarction (STEMI) 2 years earlier, with insignificant stenosis (DS of $\sim 40 \%$ ) of a proximal left anterior descending artery (LAD), and no other coronary lesions (Figure 1 A). Transthoracic echocardiography (TTE) performed at that time showed left ventricular ejection fraction (LVEF) of 35\%, which increased subsequently, and the patient was discharged without pathological Q waves in his electrocardiogram (ECG). Of note, at that time the patient was unconscious, in cardiogenic shock. Intravascular therapeutic hypothermia was initiated immediately. Current ECG showed a negative T-wave in leads V1-V6, typical for left ventricular (LV) acute ischemia and also for acute pulmonary embolism (PE). Current TTE revealed global LV hypokinesia (LVEF of $40 \%$ ) and normal right ventricle function with its correct contractility and normal intra-cavity pressure. Thus, emergent coronarography was performed as a first line intervention, prior to eventual pulmonary artery angio-computed tomography (CT). Surprisingly, the situation had not changed for 2 years, with current angiography revealing again non-obstructive proximal LAD stenosis with DS of $\sim 40 \%$ (Figure $1 \mathrm{~B}$ ). Interestingly, during early cardiac rehabilitation, typical angina symptoms occurred. Therefore, further invasive evaluation of the proximal LAD was made, with a minimal index of fractional flow reserve (FFR) measured distally of 0.77 , classifying the angiographically non-obstructive lesion as physiologically significant (Figure 1, pressure waves). Consequently, two drug-eluting stents were implanted. Significant LV contractility improvement was observed and the patient's subsequent recovery was uneventful. The presented case illustrates the clinical usefulness of FFR, extending its recognized indications to the group of patients with MINOCA [1]. It is also recommended to use IVUS for identification of a specific underlying cause of MINOCA, explicitly within the entity of epicardial coronary artery disorders (e.g. plaque rupture vs. calcified nodule vs. spontaneous dissection). Its use offers a high-resolution view with a tomographic perspective, which is in our opinion superior to FFR, especially among patients with left main or ostial lesions. Its advantage is also observed among subjects with diffusely narrowed arteries, in whom FFR measurement could be difficult to interpret and in whom spot stenting could be a potential therapeutic option. Furthermore, in these categories of patients, precise assessment of coronary anatomy and eventual use of IVUS for procedural guidance have a certain advantage over both angiography alone and angiography equipped with FFR [2].

\section{Corresponding author:}

Michał Proczka, Department of Coronary and Structural Heart Diseases, Institute of Cardiology, 42 Alpejska St, 04-628 Warsaw, Poland, phone: +48 608715 218, e-mail: michal2095@gmail.com

Received: 28.02.2018, accepted: 19.03.2018. 


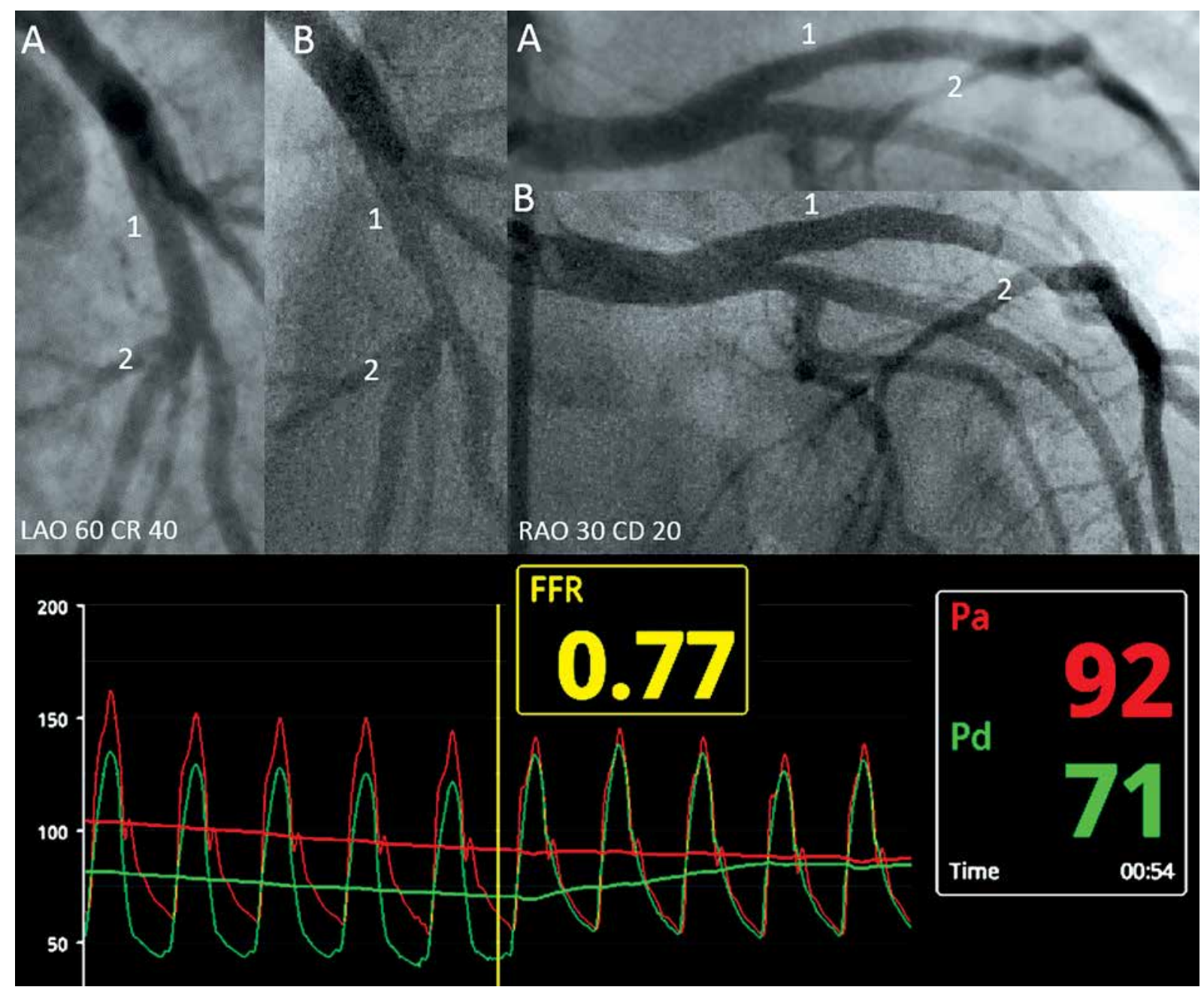

Figure 1. Previous (A) and current (B) angiographic appearances are displayed, documenting normally patent LAD (TIMI 3 and MBG 3), with insignificant stenosis (DS of $40 \%$ as measured with QCA) identified in its proximal (1) and mid segment (2, note the septal branch take-off). Minimal FFR measured during hyperemia induced with $280 \mu \mathrm{g} / \mathrm{kg} / \mathrm{min}$ adenosine i.v. was 0.77 . Note ventricularized morphology of a distal coronary pressure (Pd) wave signifying correctly induced diagnostic hyperemia, and a dicrotic notch of a proximal coronary pressure $(\mathrm{Pa})$ wave indicating correct location of the guiding catheter. During the wire pull-back both pressure readings $\mathrm{Pd}$ and Pa became ideally equal, confirming the wire signal stability and accuracy of the FFR measurement

\section{Conflict of interest}

The authors declare no conflict of intererst.

\section{References}

1. Ibanez B, James S, Agewall S, et al. 2017 ESC Guidelines for the management of acute myocardial infarction in patients presenting with ST-segment elevation. Kardiol Pol 2018; 76: 229-313.

2. Radomski M, Rychter M, Sukiennik A, Kubica J. Intermediate coronary artery stenosis - when is intervention indicated and when should it be deferred? Value of intravascular ultrasound in clinical decision making in the performance of coronary interventions. Postep Kardiol Inter 2006; 2: 294-301. 